\title{
$\underline{\text { How to design a sequential simulation (SQS) for a variety of objectives }}$
}

SM Weldon¹, M Kronfli², F Bello², R Kneebone ${ }^{2}$

1. University of Greenwich/Barts Health NHS Trust

2. Imperial College London

\section{Background/Context/Aims}

Sequential Simulation (SqS) is the physical re-enactment of longitudinal elements of care, rather than single episodes, as is currently the main format of most healthcare simulations. It takes a patient's perspective of their care pathway journey through the system rather than the healthcare professionals perspectives alone. It has been used for a variety of applications including training multi-disciplinary teams on end-of-life care, initiating integrated care, quality improvement projects, designing new models of care, evaluating new interventions, and improving care of the deteriorating patient, to name but a few.1,2 Its applications are wide, and therefore the design process can be complex. This workshop draws on an empirically and theoretically driven model, to guide participants in designing Sequential Simulations for a variety of clinical objectives.

\section{Learning Objectives and Session Description}

Learners will be split into small groups and asked to design a Sequential Simulation for a selected care pathway, using the described model. However, each group will be given a different objective for its use which will form the main basis of their design considerations. The small groups will present their design considerations to each other at the end of the session to reveal the different affordances each objective required. The learning objectives will aim to reveal the importance of objectives during the design process as well as the different considerations needed to achieve a successful Sequential Simulation. This student-centred approach aims to provide the workshop attendees with a new tool and a practical approach to designing this new concept in simulation. 


\section{Target Audiences}

Maximum number of participants Simulation educators, healthcare professionals, technical personnel. Maximum of 30 participants.

\section{REFERENCES}

1. Weldon S-M, Kneebone R, Bello F. Collaborative healthcare remodelling through Sequential Simulation (SqS): A patient and front-line staff perspective. BMJ Stel 2016; 0:1-9. doi:10.1136/bmjstel-2016-000113

2. Weldon S-M, Ralhan S, Paice E, Kneebone R, Bello F. Sequential simulation of a patient pathway. The clinical teacher 2016. doi:10.1111/tct.12549 